PROCEEDINGS OF THE

AMERICAN MATHEMATICAL SOCIETY

Volume 128, Number 5, Pages 1347-1356

S 0002-9939(99)05374-5

Article electronically published on October 18, 1999

\title{
SPLITTINGS OF BANACH SPACES INDUCED BY CLIFFORD ALGEBRAS
}

\author{
N. L. CAROTHERS, S. J. DILWORTH, AND DAVID SOBECKI
}

(Communicated by Dale Alspach)

\begin{abstract}
Let $H$ be an infinite-dimensional Hilbert space of density character $\mathfrak{m}$. By representing $H$ as a module over an appropriate Clifford algebra, it is proved that $H$ possesses a family $\left\{A_{\alpha}\right\}_{\alpha \in \mathfrak{m}}$ of proper closed nonzero subspaces such that

$$
d\left(S_{A_{\alpha}}, S_{A_{\beta}}\right)=d\left(S_{A_{\alpha}^{\perp}}, S_{A_{\beta}}\right)=d\left(S_{A_{\alpha}^{\perp}}, S_{A_{\beta}^{\perp}}\right)=\sqrt{2-\sqrt{2}} \quad(\alpha \neq \beta) .
$$

Analogous results are proved for $L_{p}$ spaces and for $c_{0}(X)$ and $\ell_{p}(X)(1 \leq p \leq$ $\infty)$ when $X$ is an arbitrary nonzero Banach space.
\end{abstract}

\section{INTRODUCTION AND NOTATION}

Let us begin with some notation. Let $C_{1}$ and $C_{2}$ be nonempty subsets of a Banach space $(X,\|\cdot\|)$. The distance between $C_{1}$ and $C_{2}$, denoted $d\left(C_{1}, C_{2}\right)$, is defined as follows:

$$
d\left(C_{1}, C_{2}\right)=\inf \left\{\left\|c_{1}-c_{2}\right\|: c_{1} \in C_{1}, c_{2} \in C_{2}\right\} .
$$

For a closed subspace $A$ of $X$, its unit sphere, denoted $S_{A}$, is the set $\{a \in A:\|a\|=$ $1\}$.

A decomposition of $X$ into the Banach space direct sum $X=A \oplus B$ of two nonzero closed subspaces $A$ and $B$ will be called a splitting of $X$, denoted $(A, B)$. For a given family $\left\{\left(A_{\gamma}, B_{\gamma}\right): \gamma \in \Gamma\right\}$ of splittings of $X$, a convenient measure of the extent to which the splittings in this family differ from one another is afforded by the quantity

$$
\delta=\inf \left\{d\left(S_{A_{\alpha}}, S_{A_{\beta}}\right), d\left(S_{B_{\alpha}}, S_{B_{\beta}}\right), d\left(S_{A_{\alpha}}, S_{B_{\gamma}}\right): \alpha, \beta, \gamma \in \Gamma \quad(\alpha \neq \beta)\right\} .
$$

If $\delta>0$, then the unit spheres of all the subspaces occurring in the splittings are separated from each other by a distance $\delta$. Such a family of splittings will be said to be well-separated.

This paper proves the existence of infinite well-separated families of splittings for certain Banach spaces. First the case of an infinite-dimensional Hilbert space $H$ is considered; here it is more natural to consider only orthogonal splittings (i.e. orthogonal decompositions) of $H$. It is proved that if $H$ has density character $\mathfrak{m}$, then there exists a family of orthogonal splittings of $H$ of cardinality $\mathfrak{m}$ for which $\delta=\sqrt{2-\sqrt{2}}$, which is best possible. The Hilbert space argument is then

Received by the editors June 19, 1998.

1991 Mathematics Subject Classification. Primary 46B20.

(C)2000 American Mathematical Society 
generalized to prove that if $X$ is an arbitrary nonzero Banach space, then $c_{0}(X)$ and $\ell_{p}(X)(1 \leq p \leq \infty)$ admit infinite well-separated families of contractively complemented splittings.

The main idea in the proof is to represent $H\left(\right.$ or $\left.\ell_{p}(X)\right)$ as a module over an appropriate infinite-dimensional Clifford algebra. The existence of the required splittings is then a consequence of algebraic identities in the Clifford algebra. The proof is self-contained and presupposes only an acquaintance with the terminology of elementary abstract algebra.

The Banach space notation and terminology employed throughout are standard. Let us only recall that the space $\ell_{p}(X)(1 \leq p \leq \infty)$ is the space of sequences $\left\langle x_{n}\right\rangle$ $\left(x_{n} \in X\right)$ equipped with the norm

$$
\left\|\left\langle x_{n}\right\rangle\right\|= \begin{cases}\left(\sum\left\|x_{n}\right\|^{p}\right)^{1 / p} & \text { for } p<\infty \\ \sup _{n}\left\|x_{n}\right\| & \text { for } p=\infty .\end{cases}
$$

The space $c_{0}(X)$ is the subspace of $\ell_{\infty}(X)$ whose elements consist of sequences which tend to zero in norm.

The proofs are valid for both real and complex Banach spaces: the underlying field of scalars (either $\mathbb{R}$ or $\mathbb{C}$ ) will be denoted by $\mathbb{F}$.

\section{Splittings of Hilbert spaces}

Let $H$ be a separable infinite-dimensional Hilbert space. For $2 \leq n \leq \infty$, define $\delta_{n}$ as follows:

$$
\delta_{n}=\sup \left\{\inf \left\{d\left(S_{A_{j}}, S_{A_{k}}\right), d\left(S_{A_{j}^{\perp}}, S_{A_{k}}\right), d\left(S_{A_{j}^{\perp}}, S_{A_{k}}\right): 0 \leq j, k<n, j \neq k\right\}\right\},
$$

where the supremum is taken over all $n$-tuples $\left\{A_{j}\right\}_{0 \leq j<n}$ of proper closed nonzero subspaces of $H$. Clearly, $\left\{\delta_{n}\right\}_{n \geq 1}$ is a decreasing sequence of nonnegative numbers with $0 \leq \delta_{\infty} \leq \lim \delta_{n}$.

We prove below (Theorem 3) that $\delta_{n}=\sqrt{2-\sqrt{2}}$ for all $2 \leq n \leq \infty$.

Proposition 1. Let $A$ be a subspace of $H$ and let $P$ be the orthogonal projection onto $A$.

(a) If $x \in S_{H}$, then

$$
\min \left\{d\left(x, S_{A}\right), d\left(x, S_{A^{\perp}}\right)\right\} \leq \sqrt{2-\sqrt{2}}
$$

with equality if and only if $\|P x\|=1 / \sqrt{2}$ (in which case $d\left(x, S_{A}\right)=d\left(x, S_{A^{\perp}}\right)=$ $\sqrt{2-\sqrt{2}}$.

(b) Let $B$ be the closed linear span of an orthonormal sequence $\left\{e_{n}\right\}$. Then

$$
d\left(S_{A}, S_{B}\right)=d\left(S_{A^{\perp}}, S_{B}\right)=\sqrt{2-\sqrt{2}}
$$

if and only if $\left\{\sqrt{2} P e_{n}\right\}$ is an orthonormal sequence in $H$.

Proof. (a) Let $a=P x$ and $a^{\prime}=(I-P) x$. Then $1=\|x\|^{2}=\|a\|^{2}+\left\|a^{\prime}\right\|^{2}$, and so $\max \left(\|a\|,\left\|a^{\prime}\right\|\right) \geq 1 / \sqrt{2}$. Without loss of generality, we shall assume that $\|a\| \geq 1 / \sqrt{2}$. Let $y \in S_{A}$. Then

$$
\|y-x\|^{2}=\|y-a\|^{2}+\left\|a^{\prime}\right\|^{2} \text {. }
$$


The distance $\|y-a\|$ is minimized when $y=a /\|a\|$; in this case, $\|y-a\|=1-\|a\|$, and so

$$
\begin{aligned}
\|y-x\|^{2} & =(1-\|a\|)^{2}+\left\|a^{\prime}\right\|^{2} \\
& =(1-\|a\|)^{2}+\left(1-\|a\|^{2}\right) \\
& =2-2\|a\| \\
& \leq 2-\sqrt{2},
\end{aligned}
$$

since we are assuming that $\|a\| \geq 1 / \sqrt{2}$. It follows that $d\left(x, S_{A}\right) \leq \sqrt{2-\sqrt{2}}$, with equality if and only if $\|a\|=\left\|a^{\prime}\right\|=1 / \sqrt{2}$.

(b) Suppose that (2) holds. Then from (a) we deduce that $d\left(x, S_{A}\right)=d\left(x, S_{A^{\perp}}\right)=$ $\sqrt{2-\sqrt{2}}$ for all $x \in S_{B}$, whence $\|P x\|=1 / \sqrt{2}$ (by (a) again) for all $x \in S_{B}$. It follows that $\sqrt{2} P$ is an isometry from $B$ into $A$, and hence that $\left\{\sqrt{2} P e_{n}\right\}$ is an orthonormal sequence in $A$. Conversely, if $\left\{\sqrt{2} P e_{n}\right\}$ is an orthonormal sequence in $A$, then $\|P x\|=1 / \sqrt{2}$ for all $x \in S_{B}$, and so (by (a)) $d\left(x, S_{A}\right)=d\left(x, S_{A^{\perp}}\right)=$ $\sqrt{2-\sqrt{2}}$ for all $x \in S_{B}$, which gives (2).

Let $\left\{e_{n}\right\}$ be an orthonormal basis for $H$, let $A$ be the closed linear span of the orthonormal sequence $\left\{e_{2 n}\right\}_{n \geq 1}$, and let $B$ be the closed linear span of the orthonormal sequence $\left\{(1 / \sqrt{2})\left(e_{2 n}+e_{2 n-1}\right)\right\}$. It follows from Proposition 1 that

$$
d\left(S_{A}, S_{B}\right)=d\left(S_{A^{\perp}}, S_{B}\right)=d\left(S_{A}, S_{B^{\perp}}\right)=d\left(S_{A^{\perp}}, S_{B^{\perp}}\right)=\sqrt{2-\sqrt{2}},
$$

and also that this pair of splittings is the best possible in the sense that the constant $\sqrt{2-\sqrt{2}}$ cannot be improved (i.e., increased). This proves that $\delta_{2}=\sqrt{2-\sqrt{2}}$.

In fact, (3) determines $A$ and $B$ uniquely up to an isomorphism of $H$, as the following result shows.

Corollary 2. Suppose that $H$ is a separable infinite-dimensional Hilbert space. Let $A$ and $B$ be closed subspaces of $H$ which satisfy equality (3). Then there exist orthonormal bases $\left\{e_{n}\right\}$ and $\left\{f_{n}\right\}$ of $A$ and $A^{\perp}$ such that $\left\{(1 / \sqrt{2})\left(e_{n}+f_{n}\right)\right\}$ and $\left\{(1 / \sqrt{2})\left(e_{n}-f_{n}\right)\right\}$ are orthonormal bases of $B$ and $B^{\perp}$.

Proof. Let $P$ be the orthogonal projection of $H$ onto $A$ and let $Q=I-P$. Let $\left\{g_{n}\right\}$ be an orthonormal basis for $B$. Then, from Proposition 1 and (3), it follows that $\left\{e_{n}\right\}$ and $\left\{f_{n}\right\}$ are orthonormal sequences in $A$ and $A^{\perp}$, where $e_{n}=(\sqrt{2}) P\left(g_{n}\right)$ and $f_{n}=(\sqrt{2}) Q\left(g_{n}\right)$. Let us show that $\left\{e_{n}\right\}$ is in fact an orthonormal basis of $A$. So suppose that $e \in A$ and that $\left\langle e, e_{n}\right\rangle=0$ for all $n$. Then $\langle e, P(y)\rangle=0$ for all $y \in B$. From Proposition 1 and (3) we have $e=y+z$ where $y \in B, z \in B^{\perp}$, and $\|y\|=\|z\|=(1 / \sqrt{2})\|e\|$. Hence $e=P(e)=P(y)+P(z)$, and so

$$
\frac{\|e\|^{2}}{4}=\|P(z)\|^{2}=\|e\|^{2}+\|P(y)\|^{2}=\|e\|^{2}+\frac{\|e\|^{2}}{4} .
$$

Thus $e=0$, which implies that $\left\{e_{n}\right\}$ is an orthonormal basis of $A$. Similarly $\left\{f_{n}\right\}$ is an orthonormal basis of $A^{\perp}$. Note that $g_{n}=(1 / \sqrt{2})\left(e_{n}+f_{n}\right)$ and recall that $\left\{g_{n}\right\}$ is an orthonormal basis of $B$. Clearly, $\left\{(1 / \sqrt{2})\left(e_{n}-f_{n}\right)\right\}$ is an orthonormal basis of $B^{\perp}$. 
Theorem 3. Let $H$ be a separable infinite-dimensional Hilbert space. There exists a sequence $\left\{A_{n}\right\}$ of subspaces of $H$ such that

$$
d\left(S_{A_{n}}, S_{A_{m}}\right)=d\left(S_{A_{n}}, S_{A_{m}^{\perp}}\right)=d\left(S_{A_{n}^{\perp}}, S_{A_{m}^{\perp}}\right)=\sqrt{2-\sqrt{2}} \quad(n \neq m) .
$$

In particular, $\delta_{n}=\sqrt{2-\sqrt{2}}$ for all $2 \leq n \leq \infty$.

Proof. We begin our construction by defining a group $G$ which is generated by an element -1 and by the sequence of symbols $\left\{e_{n}\right\}_{n \geq 1}$ satisfying the generating relations:

$$
e_{n}^{2}=-1 \quad \text { and } \quad(-1)^{2}=1 .
$$

The element -1 belongs to the center of $G$ and we define for any $g \in G$ :

$$
-g=(-1)(g)=(g)(-1) .
$$

Multiplication among the $e_{n}$ 's is defined to be anti-commutative:

$$
e_{m} e_{n}=-e_{n} e_{m} \quad(n \neq m) .
$$

The elements of $G$ consist of finite strings of the generators. For any finite subset $I \subset \mathbb{N}$, let $e_{I}=e_{n_{1}} e_{n_{2}} \cdots e_{n_{k}}$, where $I=\left\{n_{1}, n_{2}, \ldots, n_{k}\right\}$ and $1 \leq n_{1}<n_{2}<\cdots<$ $n_{k}$, and let $e_{I}=1$ for $I=\emptyset$. Finally, setting $W=\left\{e_{I}: I\right.$ is a finite subset of $\left.\mathbb{N}\right\}$, we can list the group elements thus:

$$
G=\{w,-w: w \in W\} .
$$

Consider the collection $C$ of all finite sums of the form $\sum_{w \in W} \lambda_{w} w$, where $\lambda_{w} \in \mathbb{F}$. Next define scalar multiplication on $G \backslash W=\{-w: w \in W\}$ as one would expect:

$$
\lambda(-w)=(-\lambda) w \quad(w \in W, \lambda \in \mathbb{F}) .
$$

Then $C$ becomes an algebra over $\mathbb{F}$ with multiplication defined in the obvious way using the group multiplication of $G$ and the distributive law. In fact, if we denote by $V$ the inner product space over $\mathbb{F}$ which has orthonormal basis $\left\{e_{n}\right\}$, then $C$ is the universal Clifford algebra associated to $V$. We refer the reader to [3] for a discussion of Clifford algebras and for a proof of the associativity of the algebra multiplication.

Let the symbols $x$ and $y$ be the generators of a free left-module over $C$, which we shall denote by $M$. It follows that every $m \in M$ is uniquely expressible in the form $c_{1} x+c_{2} y$, where $c_{1}, c_{2} \in C$. Note that the elements of $M$ are of the form

$$
m=\left(\sum_{w \in W} \lambda_{w} w\right) x+\left(\sum_{w \in W} \mu_{w} w\right) y=\sum_{w \in W}\left(\lambda_{w}\right) w x+\sum_{w \in W}\left(\mu_{w}\right) w y,
$$

where the sums are finite and the $\lambda$ 's and $\mu$ 's belong to $\mathbb{F}$. Next we turn the module $M$ into an inner product space over $\mathbb{F}$ by taking the set $\Xi=\{w x, w y: w \in W\}$ to be an orthonormal spanning set. Let $H$ be the completion of this inner product space, so that $H$ is a separable infinite-dimensional Hilbert space with orthonormal basis $\Xi$. Note that the inner product $\langle$,$\rangle on H$ is defined by the relations

$$
\left\langle w x, w^{\prime} y\right\rangle=0 \quad\left(w, w^{\prime} \in W\right)
$$

and

$$
\left\langle w x, w^{\prime} x\right\rangle=\left\langle w y, w^{\prime} y\right\rangle= \begin{cases}1, & \text { for } w=w^{\prime} \\ 0, & \text { for } w \neq w^{\prime}\end{cases}
$$


For each $w^{\prime} \in W$ there exists a permutation $\pi_{w^{\prime}}$ of $W$ such that the mapping $w \mapsto w^{\prime} w$ is given by

$$
w^{\prime} w= \pm \pi_{w^{\prime}}(w)
$$

where the choice of signs depends, of course, on the element $w$. For convenience, let us call such a mapping a sign-changing permutation. It follows that the linear operator corresponding to the action of $w^{\prime} \in W$ on $H$ given by

$$
w^{\prime}\left\{\sum_{w}\left(\lambda_{w} w x+\gamma_{w} w y\right)\right\}=\sum_{w}\left\{\lambda_{w}\left(w^{\prime} w x\right)+\gamma_{w}\left(w^{\prime} w y\right)\right\}
$$

is in fact an isomorphism of $H$, as it is the linear extension of a sign-changing permutation of the orthonormal basis $\Xi$. We may now regard $H$ as a left $C$-module by extending the action of (4) to the whole of $C$ by linearity.

Let $A_{n}$ be the norm-closed left $C$-submodule generated by the element $x+e_{n} y$. One easily sees that

$$
\Xi_{n}=\left\{\frac{w x+\left(w e_{n}\right) y}{\sqrt{2}}: w \in W\right\}
$$

is an orthonormal basis for $A_{n}$, and that

$$
\Xi_{n}^{\prime}=\left\{\frac{w x-\left(w e_{n}\right) y}{\sqrt{2}}: w \in W\right\}
$$

is an orthonormal basis for $A_{n}^{\perp}$. Having defined the subspaces that make up our sequence of splittings, it remains to show that the distances $d\left(S_{A_{n}}, S_{A_{m}}\right), d\left(S_{A_{n}}, S_{A_{m}^{\perp}}\right)$ and $d\left(S_{A_{n}^{\perp}}, S_{A_{m}^{\perp}}\right)$ are all equal to $\sqrt{2-\sqrt{2}}$ for $n \neq m$.

Let $P_{n}$ denote the orthogonal projection from $H$ onto $A_{n}$. To prove that $d\left(S_{A_{n}}, S_{A_{m}}\right)=\sqrt{2-\sqrt{2}}$, we shall invoke Proposition 1. It suffices to prove that $P_{n}$ maps the basis $\Xi_{m}$ of $A_{m}$ onto an orthogonal sequence of vectors in $A_{n}$, all having norm $1 \sqrt{2}$. To this end, let us express the vector $\left(x+e_{m} y\right) / \sqrt{2}$ in the form

$$
\frac{x+e_{m} y}{\sqrt{2}}=\left(\frac{1-e_{m} e_{n}}{2}\right)\left(\frac{x+e_{n} y}{\sqrt{2}}\right)+\left(\frac{1+e_{m} e_{n}}{2}\right)\left(\frac{x-e_{n} y}{\sqrt{2}}\right) .
$$

Then, for $w \in W$, we have

$$
\frac{w x+\left(w e_{m}\right) y}{\sqrt{2}}=\left(\frac{w\left(1-e_{m} e_{n}\right)}{2}\right)\left(\frac{x+e_{n} y}{\sqrt{2}}\right)+\left(\frac{w\left(1+e_{m} e_{n}\right)}{2}\right)\left(\frac{x-e_{n} y}{\sqrt{2}}\right) .
$$

Note that in this form the vector $\left(w x+\left(w e_{m}\right) y\right) / \sqrt{2}$ is written as the sum of vectors from $A_{n}$ and $A_{n}^{\perp}$, and so it is easy to compute its projection onto $A_{n}$ :

$$
\begin{aligned}
P_{n}\left(\frac{w x+\left(w e_{m}\right) y}{\sqrt{2}}\right) & =\frac{w\left(1-e_{m} e_{n}\right)}{2} \cdot \frac{\left(x+e_{n} y\right)}{\sqrt{2}} \\
& =\frac{1}{2 \sqrt{2}}\left\{w x-\left(w e_{m} e_{n}\right) x+\left(w e_{n}\right) y-\left(w e_{m} e_{n} e_{n}\right) y\right\} \\
& =\frac{1}{2 \sqrt{2}}\left\{w x-\left(w e_{m} e_{n}\right) x+\left(w e_{n}\right) y+\left(w e_{m}\right) y\right\} .
\end{aligned}
$$


First observe that from (5)

$$
\left\|P_{n}\left(\frac{w x+\left(w e_{m}\right) y}{\sqrt{2}}\right)\right\|=\frac{1}{2 \sqrt{2}}\left(1^{2}+1^{2}+1^{2}+1^{2}\right)^{1 / 2}=\frac{1}{\sqrt{2}},
$$

which proves that $P_{n}$ maps each member of $\Xi_{m}$ onto a vector of norm $1 / \sqrt{2}$.

Now let us show that $P_{n}$ maps $\Xi_{m}$ onto an orthogonal sequence; that is, that

$$
\left\langle P_{n}\left(\frac{w x+\left(w e_{m}\right) y}{\sqrt{2}}\right), P_{n}\left(\frac{w^{\prime} x+\left(w^{\prime} e_{m}\right) y}{\sqrt{2}}\right)\right\rangle=0
$$

for all $w \neq w^{\prime}$.

Replacing $w$ by $w^{\prime}$ in (5), we get

$$
P_{n}\left(\frac{w^{\prime} x+\left(w^{\prime} e_{m}\right) y}{\sqrt{2}}\right)=\frac{1}{2 \sqrt{2}}\left\{w^{\prime} x-\left(w^{\prime} e_{m} e_{n}\right) x+\left(w^{\prime} e_{n}\right) y+\left(w^{\prime} e_{m}\right) y\right\} .
$$

The four vectors occurring on the right-hand side of (5) and the four on the right-hand side of (6) belong to the orthonormal basis $\Xi$ of $H$. If these sets of basis vectors are disjoint, then clearly the inner product is zero. These sets of basis vectors will be disjoint unless

$$
w^{\prime}= \pm w e_{m} e_{n} \quad \text { or } \quad w= \pm w^{\prime} e_{m} e_{n} .
$$

It remains to show that the inner product is zero if one of the two conditions of (7) holds. Without loss of generality, let us assume that $w^{\prime}=w e_{m} e_{n}$. In this case, we have

$$
\begin{aligned}
w^{\prime} x- & \left(w^{\prime} e_{m} e_{n}\right) x+\left(w^{\prime} e_{n}\right) y+\left(w^{\prime} e_{m}\right) y \\
& =\left(w e_{m} e_{n}\right) x-\left(w e_{m} e_{n} e_{m} e_{n}\right) x+\left(w e_{m} e_{n} e_{n}\right) y+\left(w e_{m} e_{n} e_{m}\right) y \\
& =\left(w e_{m} e_{n}\right) x-\left(w e_{m}\left(-e_{m} e_{n}\right) e_{n}\right) x+\left(w e_{m}(-1)\right) y+\left(w e_{m}\left(-e_{m} e_{n}\right)\right) y \\
& =\left(w e_{m} e_{n}\right) x+(w(-1)(-1)) x-\left(w e_{m}\right) y-\left(w(-1) e_{n}\right) y \\
& =\left(w e_{m} e_{n}\right) x+w x-\left(w e_{m}\right) y+\left(w e_{n}\right) y,
\end{aligned}
$$

and it follows from (6) that

$$
P_{n}\left(\frac{w^{\prime} x+\left(w^{\prime} e_{m}\right) y}{\sqrt{2}}\right)=\frac{1}{2 \sqrt{2}}\left\{w x+\left(w e_{m} e_{n}\right) x-\left(w e_{m}\right) y+\left(w e_{n}\right) y\right\} .
$$

Computing the inner product, we get

$$
\begin{aligned}
& \left\langle P_{n}\left(\frac{w x+\left(w e_{m}\right) y}{\sqrt{2}}\right), P_{n}\left(\frac{w^{\prime} x+\left(w^{\prime} e_{m}\right) y}{\sqrt{2}}\right)\right\rangle \\
& =\frac{1}{8}\left\langle w x-\left(w e_{m} e_{n}\right) x+\left(w e_{m}\right) y+\left(w e_{n}\right) y,\right. \\
& \left.w x+\left(w e_{m} e_{n}\right) x-\left(w e_{m}\right) y+\left(w e_{n}\right) y\right\rangle \\
& =\frac{1}{8}\left\{\langle w x, w x\rangle-\left\langle\left(w e_{m} e_{n}\right) x,\left(w e_{m} e_{n}\right) x\right\rangle\right. \\
& \left.=\frac{1}{8}\left\{\|w x\|^{2}-\|\left(w e_{m}\right) y,\left(w e_{m}\right) y\right\rangle+\left\langle\left(w e_{n}\right) y,\left(w e_{n}\right) y\right\rangle\right\} \\
& =\frac{1}{8}\{1-1-1+1\}=0 .
\end{aligned}
$$


Thus, $P_{n}$ maps $\Xi_{m}$ onto an orthogonal sequence of vectors in $A_{n}$, which completes the proof that $d\left(S_{A_{n}}, S_{A_{m}}\right)=\sqrt{2-\sqrt{2}}$. Similar computations prove that $d\left(S_{A_{n}}, S_{A_{m}^{\perp}}\right)$ and $d\left(S_{A_{n}^{\perp}}, S_{A_{m}^{\perp}}\right)$ are also equal to $\sqrt{2-\sqrt{2}}$.

The proof of Theorem 3 readily extends to nonseparable Hilbert spaces to yield the result stated in the abstract (or a paraphrase thereof).

Theorem 4. Let $\Gamma$ be an infinite set. Then there exists a family $\left\{A_{\gamma}\right\}_{\gamma \in \Gamma}$ of subspaces of $\ell_{2}(\Gamma)$ such that

$$
d\left(S_{A_{\gamma}}, S_{A_{\delta}}\right)=d\left(S_{A_{\gamma}}, S_{A_{\delta}^{\perp}}\right)=d\left(S_{A_{\gamma}^{\perp}}, S_{A_{\delta}^{\perp}}\right)=\sqrt{2-\sqrt{2}}
$$

for all $\gamma, \delta \in \Gamma$ with $\gamma \neq \delta$.

Proof. The proof is analogous to the proof of Theorem 3, with the modification that the group $G$ is now generated by the (possibly uncountable) set $\left\{e_{\gamma}\right\}_{\gamma \in \Gamma}$. We construct the Clifford algebra $C$ that is associated to the inner product space which has $\left\{e_{\gamma}\right\}_{\gamma \in \Gamma}$ as an orthonormal basis. Next we define the $C$-module $M$ and the Hilbert space $H$, as in the proof of Theorem 3 , and one sees that $H$ has an orthonormal spanning set of the same cardinality as $\Gamma$. Then we define a collection of subspaces $A_{\gamma}(\gamma \in \Gamma)$, as in the proof of Theorem 3. The distance calculations are identical.

Remark 5. Obviously, the cardinality of $\Gamma$ is the largest possible cardinality of any well-separated family of splittings of $\ell_{2}(\Gamma)$.

\section{Splittings of Banach spaces}

Recall that there exists an infinite-dimensional Banach space $X$ [1] which is hereditarily indecomposable; that is, $X$ has the property that no closed subspace of $X$ can be expressed as a direct sum of two further infinite-dimensional closed subspaces. Let $X=A_{1} \oplus B_{1}$ and $X=A_{2} \oplus B_{2}$ be a pair of splittings of $X$ such that both $A_{1}$ and $A_{2}$ are infinite-dimensional. Since $X$ is indecomposable, both $B_{1}$ and $B_{2}$ are finite-dimensional, whence $A_{1} \cap A_{2}$ is nonzero, and in particular $d\left(S_{A_{1}}, S_{A_{2}}\right)=0$. This shows that Theorem 3 cannot be extended to the category of Banach spaces simply by replacing orthogonal projections by bounded projections.

However, we are able to modify our construction in order to obtain a result for $\ell_{p}(X)$, when $X$ is an arbitrary nonzero Banach space. Regarding $X$ and $M$ as vector spaces over $\mathbb{F}$, we may form the vector space tensor product $X \otimes M$. A typical element of $X \otimes M$ may be expressed uniquely as a finite sum of the form:

$$
a=\sum_{w \in W}\left\{a_{w} \otimes(w x)+a_{w}^{\prime} \otimes(w y)\right\} \quad\left(a_{w}, a_{w}^{\prime} \in X\right) .
$$

We now equip $X \otimes M$ with the norm

$$
\left|\|a \mid\|_{p}= \begin{cases}\left(\sum\left\|a_{w}\right\|^{p}+\left\|a_{w}^{\prime}\right\|^{p}\right)^{1 / p} & \text { for } p<\infty, \\ \sup \left\{\left\|a_{w}\right\|,\left\|a_{w}^{\prime}\right\|: w \in W\right\} & \text { for } p=\infty .\end{cases}\right.
$$

Taking the completion of $\left|\|\cdot \mid\|_{p}\right.$ we obtain, for $p<\infty$, a Banach space $X_{p}$ that is isometrically isomorphic to $\ell_{p}(X)$, and, for $p=\infty$, a space $X_{\infty}^{0}$ that is isometrically isomorphic to $c_{0}(X)$. The elements of $X_{p}$ have the unique representation given by (8) when the sums are allowed to be infinite. 
Similarly, one defines the space $X_{\infty}$, which is isometrically isomorphic to $\ell_{\infty}(X)$, in the obvious fashion.

The action of the Clifford algebra $C$ on $M$ extends to $X \otimes M$ by means of the following definition:

$$
c\left(\sum\left\{a_{w} \otimes(w x)+a_{w}^{\prime} \otimes(w y)\right\}\right)=\sum\left\{a_{w} \otimes(c w x)+a_{w}^{\prime} \otimes(c w y)\right\} .
$$

Extending this action to $X_{p}$ (by continuity) turns these Banach spaces into $C$ modules for which each $w \in W$ acts as an isometric isomorphism of $X_{p}$.

We can now define, for each $n \geq 1$, a pair of complementary subspaces in $X_{p}$. Let $A_{n}$ consist of all vectors $a \in X_{p}$ of the form

$$
a=\sum_{w \in W} a_{w} \otimes\left(w x+w e_{n} y\right),
$$

and let $B_{n}$ consist of all vectors $b$ of the form

$$
b=\sum_{w \in W} b_{w} \otimes\left(w x-w e_{n} y\right) .
$$

Proposition 6. Let $1 \leq p \leq \infty$. Then $A_{n}$ and $B_{n}$ are contractively complemented in $X_{p}$ and

$$
d\left(S_{A_{n}}, S_{A_{m}}\right)=d\left(S_{A_{n}}, S_{B_{m}}\right)=d\left(S_{B_{n}}, S_{B_{m}}\right) \geq \frac{1}{2} \quad(m \neq n) .
$$

The same result holds for $X_{\infty}^{0}$.

Proof. We give the proof only for $p<\infty$. Fix $m, n \in \mathbb{N}$. Consider the projection $P_{n}$ on $X_{p}$ given by

$$
P_{n}(a)=\sum_{w \in W}\left((1 / 2) a_{w}+(1 / 2) a_{w}^{\prime}\right) \otimes\left(w x+w e_{n} y\right),
$$

when $a$ is represented (uniquely) as:

$$
a=\sum_{w \in W}\left\{a_{w} \otimes(w x)+a_{w}^{\prime} \otimes\left(w e_{n} y\right)\right\} \quad\left(a_{w}, a_{w}^{\prime} \in X\right) .
$$

Clearly $A_{n}$ is the range of $P_{n}$, and $P_{n}$ is contractive by convexity of the norm in $X$. The complementary projection $Q_{n}=I-P_{n}$ is also contractive and has range $B_{n}$. Suppose that $m \neq n$. Observe that $W$ can be expressed as the disjoint union $W=W_{1} \cup W_{2}$, in which each $w \in W_{1}$ corresponds to a unique element $w^{\prime}= \pm w e_{m} e_{n}$ in $W_{2}$. Thus each $a \in A_{m}$ can be written uniquely in the form

$$
a=\sum_{w \in W_{1}}\left\{a_{w} \otimes\left(w\left(x+e_{m} y\right)\right)+b_{w} \otimes\left(\left(w e_{m} e_{n}\right)\left(x+e_{m} y\right)\right)\right\} .
$$

From the identity

$$
w x+\left(w e_{m}\right) y=\left(\frac{w\left(1-e_{m} e_{n}\right)}{2}\right)\left(x+e_{n} y\right)+\left(\frac{w\left(1+e_{m} e_{n}\right)}{2}\right)\left(x-e_{n} y\right),
$$


we obtain

$$
\begin{aligned}
P_{n}(a)= & \sum_{w \in W_{1}} a_{w} \otimes\left(\frac{w\left(1-e_{m} e_{n}\right)}{2}\right)\left(x+e_{n} y\right) \\
& +\sum_{w \in W_{1}} b_{w} \otimes\left(\frac{w e_{m} e_{n}\left(1-e_{m} e_{n}\right)}{2}\right)\left(x+e_{n} y\right) \\
= & \sum_{w \in W_{1}}\left\{\left(\frac{a_{w}+b_{w}}{2}\right) \otimes w\left(x+e_{n} y\right)+\left(\frac{b_{w}-a_{w}}{2}\right) \otimes w e_{m} e_{n}\left(x+e_{n} y\right)\right\},
\end{aligned}
$$

whence

$$
\begin{aligned}
\left|\left\|P_{n}(a)\right\|\right|^{p} & =\sum_{w \in W_{1}}\left\{\left\|(1 / 2)\left(a_{w}+b_{w}\right)\right\|^{p}+\left\|(1 / 2)\left(a_{w}-b_{w}\right)\right\|^{p}\right\} \\
& \geq 2^{-p} \sum_{w \in W_{1}}\left\{\left\|a_{w}\right\|^{p}+\left\|b_{w}\right\|^{p}\right\} \\
& =2^{-p}|\|a\||^{p},
\end{aligned}
$$

and so $\left|\left\|P_{n}(a)\right\|\right| \geq(1 / 2)|\|a\||$. A similar calculation shows that $\left|\left\|Q_{n}(a)\right\|\right| \geq$ $(1 / 2) \mid\|a\| \|$. Finally,

$$
d\left(a, S_{A_{n}}\right) \geq\left|\left\|Q_{n}(a)\right\|\right| \geq(1 / 2)|\|a\||,
$$

which implies that $d\left(S_{A_{m}}, S_{A_{n}}\right) \geq 1 / 2$. By symmetry, it follows that

$$
d\left(S_{B_{m}}, S_{A_{n}}\right)=d\left(S_{B_{m}}, S_{B_{n}}\right)=d\left(S_{A_{m}}, S_{A_{n}}\right) .
$$

Since $X_{p}$ is isometrically isomorphic to $\ell_{p}(X)$, we obtain the following theorem.

Theorem 7. Let $1 \leq p<\infty$ and let $X$ be an arbitrary nonzero Banach space. For each $n \geq 1$, there exist closed subspaces $A_{n}$ and $B_{n}$ of $\ell_{p}(X)$ such that the following hold:

(a) $\ell_{p}(X)=A_{n} \oplus B_{n}$ and the corresponding projections are contractions;

(b) $d\left(S_{A_{n}}, S_{A_{m}}\right)=d\left(S_{A_{n}}, S_{B_{m}}\right)=d\left(S_{B_{n}}, S_{B_{m}}\right) \geq 1 / 2$ for $m \neq n$.

The same result holds for $c_{0}(X)$ and $\ell_{\infty}(X)$.

Next we consider splittings of Lebesgue $L_{p}$ spaces for which we require the following lemma, whose proof is an easy deduction from Clarkson's inequalities (see e.g. [4), which we leave to the reader.

Lemma 8. Let $(X, \Sigma, \mu)$ be a measure space and let $1<p<\infty$. For all $f, g \in$ $L_{p}(\mu)$, we have

$$
\left(\left\|\frac{f+g}{2}\right\|^{p}+\left\|\frac{f-g}{2}\right\|^{p}\right) \geq 2^{-p / p^{*}}\left(\|f\|^{p}+\|g\|^{p}\right),
$$

where $p^{*}=\min (p, p /(p-1))$.

Theorem 9. Let $(X, \Sigma, \mu)$ be a separable $\sigma$-finite measure space which has either no atoms or infinitely many atoms and let $1<p<\infty$. For each $n \geq 1$, there exist closed subspaces $A_{n}$ and $B_{n}$ of $L_{p}(\mu)$ such that the following hold:

(a) $L_{p}(\mu)=A_{n} \oplus B_{n}$ and the corresponding projections are contractions;

(b) $d\left(S_{A_{n}}, S_{A_{m}}\right)=d\left(S_{A_{n}}, S_{B_{m}}\right)=d\left(S_{B_{n}}, S_{B_{m}}\right) \geq 2^{-1 / p^{*}}$ for $m \neq n$, where $p^{*}=\min (p, p /(p-1))$.

The same result holds for $L_{1}(\mu)$ and $L_{\infty}(\mu)$ with constant $1 / 2$. 
Proof. The assumption on $(S, \Sigma, \mu)$ implies that $L_{p}(\mu)$ is isometrically isomorphic to $\ell_{p}\left(L_{p}(\mu)\right)$. (In fact $L_{p}(\mu)$ will be isometrically isomorphic to either $\ell_{p}, L_{p}(0,1)$ or $\ell_{p} \oplus_{p} L_{p}(0,1)$.) Using Lemma 8 we can replace the factor of $2^{-p}$ in equation (9) of Proposition 6 by the larger constant of $2^{-p / p^{*}}$. With this change the argument of Proposition 6 yields the desired result.

Remark 10. The constant $2^{-1 / p^{*}}$ is probably not best possible. In fact, we have already seen that for $p=2$ the best constant is $\sqrt{2-\sqrt{2}}=0.7653 \ldots$ (as opposed to $1 / \sqrt{2}=0.7071 \ldots)$. Finally, let us observe one further consequence.

Corollary 11. Let $K$ be an infinite compact metric space. Then $C(K)$ admits an infinite well-separated family of splittings.

Proof. This follows from Theorem 7 since $C(K)$ is linearly isomorphic to $c_{0}(C(K))$ whenever $K$ is an infinite compact metric space (see e.g. 4]).

\section{ACKNOWLEDGEMENT}

The authors thank Ralph Howard for the question which began this project.

\section{REFERENCES}

1. W. T. Gowers and B. Maurey, The unconditional basic sequence problem, J. Amer. Math. Soc. 6 (1993), 851-874. MR 94k:46021

2. Edwin Hewitt and Karl Stromberg, Real and Abstract Analysis, Springer-Verlag, New York, 1965. MR 32:5826

3. Serge Lang, Algebra, Addison-Wesley, Reading, MA, 1965. MR 33:5416

4. J. Lindenstrauss and L. Tzafriri, Classical Banach spaces, Lecture Notes in Math. Vol. 338, Springer-Verlag, Berlin-Heidelberg-New York, 1973. MR 55:3344

Department of Mathematics, Bowling Green State University, Bowling Green, Ohio 43402

E-mail address: carother@math.bgsu.edu

Department of Mathematics, University of South Carolina, Columbia, South CarOLINA 29208

E-mail address: dilworth@math.sc.edu

Department of Mathematics, Miami University, Hamilton, Ohio 45014

E-mail address: sobeckdm@muohio.edu 